\title{
Louis-Charles Lefebvre de Bellefeuille, prêtre de Saint-Sulpice, 1795-1838
}

\section{J.-Bruno Harel}

Volume 49, 1982

URI : https://id.erudit.org/iderudit/1007088ar

DOI : https://doi.org/10.7202/1007088ar

Aller au sommaire du numéro

Éditeur(s)

Les Éditions Historia Ecclesiæ Catholicæ Canadensis Inc.

ISSN

0318-6172 (imprimé)

1927-7067 (numérique)

Découvrir la revue

Citer cet article

Harel, J.-B. (1982). Louis-Charles Lefebvre de Bellefeuille, prêtre de

Saint-Sulpice, 1795-1838. Sessions d'étude - Société canadienne d'histoire de

l'Église catholique, 49, 7-24. https://doi.org/10.7202/1007088ar

Tous droits réservés @ Les Éditions Historia Ecclesiæ Catholicæ Canadensis Inc., 1982
Ce document est protégé par la loi sur le droit d'auteur. L'utilisation des services d'Érudit (y compris la reproduction) est assujettie à sa politique d'utilisation que vous pouvez consulter en ligne.

https://apropos.erudit.org/fr/usagers/politique-dutilisation/ 


\section{Louis-Charles Lefebvre de Bellefeuille, prêtre de Saint-Sulpice,} 1795-1838

\section{UN MARIAGE}

À la fin du XVIII ${ }^{\mathrm{e}}$ siècle, le village de Saint-Eustache regroupait, sur la rive nord de la rivière des Mille-Iles (rivière Jésus), sur les deux berges de la petite rivière du Chesne, une centaine de maisons occupées par quelque 600 personnes. L'agglomération constituait le principal centre de la partie ouest de la Seigneurie des Mille-Isles, partie plus souvent appelée le Fief Dumont ou de la Rivière du Chesne. La Seigneurie connaissait un accroissement considérable, l'île Jésus saturée ne fournissait plus aux jeunes de bonnes terres. La Seigneurie du lac des Deux-Montagnes ouverte à la colonisation en $\mathbf{1 7 8 0}$ profitait aussi de ce phénomène social. À Saint-Eustache, la géographie offrait une pointe avançant entre les deux rivières. Le coeur du village s'y était installé. On y trouvait de la place pour une promenade bordée au sud-ouest par le premier presbytère de bois (perpendiculaire à la rivière principale), l'église et le cimetière, et à l'est la maison du seigneur Dumont. L'église avait belle allure avec ses 120 pieds de long sur 48 de large et des murs de pierre de taille de 20 pieds de haut. Une sacristie à l'arrière complétait le lieu du culte. Un modeste clocher sur le faîte du fronton triangulaire de la façade donnait encore plus d'assurance au bâtiment. Enfin l'esplanade se terminait à l'est par la demeure seigneuriale bâtie en pierre de taille sur deux étages. ${ }^{1}$

C'est dans le cadre tranquille, coquet, revêtu du manteau de l'hiver que le mardi, 5 février $1793,{ }^{2}$ s'animait une foule disparate attiree par un événement hors de l'ordinaire. Le seigneur, Eustache Lambert dit

${ }^{1}$ L.-Adolphe Huguet-Latour, Annuaire de Ville-Marie, origine, utilité et progrès des Institutions catholiques de Montréal, Montréal, Eusèbe Sénécal, 1864, p. 134-141.

2 Paroisse de Saint-Eustache, Registre des baptêmes, mariages et sépultures de 1793, f5, recto. 
Dumont, mariait sa fille à un parti fort intéressant, unissant ainsi deux grandes familles. En effet Louise-Angélique Lambert dite Dumont, fille de Louis-Eustache Lambert-Dumont et de Marguerite Boisseau, épousait Antoine Lefebvre de Bellefeuille, écuyer, grand voyer du district des Trois-Rivières, l'un des juges de ce district, fils de François Lefebvre de Bellefeuille, seigneur de Grand Pabos en Gaspésie et de MarieJosette Hertel de Cournoyer. Le registre des mariages nous indique que le curé Benjamin-Nicolas Maillou, qui se dit cousin de l'époux, a autorisé Louis Poulin-Cressé de Courval, cousin germain de la mariée à requérir les consentements et à donner la bénédiction nuptiale. Les fiancés s'étaient sans doute connus aux Trois-Rivières. Les parents de la mariée y habitaient habituellement en 1770 et, en 1781 , le père avait cinq soeurs qui y résidaient. Quant à la famille Lefebvre de Bellefeuille, elle s'y était établie après la conquête. Le registre des mariages nous révèle que les parents des Trois-Rivières assistaient à l'événement. On y voit les Hertel de Cournoyer, les Boucher de Niverville, les de Bellefeuille et les Dumont, les Poulin-Cressé de Courval, les Nolan Lamarque et quelques habitants de Saint-Eustache. Ce mariage attirait les amis, les censitaires curieux et je ne doute pas que tous les témoins formulaient des voeux de bonheur, présages d'une union heureuse et féconde pour l'avenir.

Les souhaits formulés par les assistants du mariage de février 1793 se réalisèrent rapidement. Les nouveaux époux qui habitèrent le manoir de l'esplanade de Saint-Eustache virent leur foyer s'enrichir de dix enfants, une fille et neuf garçons. ${ }^{3}$ Mais trois moururent en bas âge, plusieurs disparurent entre vingt et quarante-cinq ans et un seul mourut octogénaire. Les garçons étudièrent au Collège de Montréal, trois choisirent le droit et devinrent notaires, deux choisirent l'état religieux et furent des prêtres zélés.

Le père, mort à l'âge de 61 ans, le 8 juin 1816 , fut inhumé sous l'église. Son épouse lui survécut 15 ans, mourut le 24 novembre 1831 à 65 ans. Elle fut enterrée aussi sous l'église.

${ }^{3}$ Voici les dix enfants d'Antoine Lefebvre de Bellefeuille (1755-1816) et de LouiseAngélique Lambert dite Dumont (1766-1831) : Eustache-Antoine (1793-1836), LouisCharles (1795-1838), François-Louis (1797-1836), Édouard-Louis (1797-1815), Marguerite-Angélique (1798-1822), Henri-Nicolas (1800-1825), Eustache-Prosper (mars-août 1801), Joseph (1803-1887), Jean-Baptiste (1804-1805), Grégoire (mai-juillet 1806). Sur la famille Lefebvre de Bellefeuille, voir A.C. de LERY MACDONALD, "La famille Lefebvre de Bellefeuille», dans Revue Canadienne, t. IV, 1884, pp. 168-176, 235-247, 291-302; et François DANIEL, p.S.S., Histoire des Grandes Familles Françaises du Canada, Montréal, Eusèbe Sénécal, 1867. 


\section{ÉDUCATION PRIMAIRE ET SECONDAIRE}

On ne sait pas exactement quels furent les professeurs des aînés de la famille Lefebvre de Bellefeuille. Les parents ont pu jouer ce rôle quelque temps. Le curé B.-N. Maillou, chargé de la paroisse SaintEustache de 1791 à 1810 , a peut-être aussi contribué à cette tâche.

Mais il est plus'probable cependant que les aînés profitèrent de l'enseignement de Louis-Généreux Labadie (1765-1824), un laïc qui consacra sa vie à l'éducation primaire dans diverses paroisses du Québec. Le curé et les paroissiens de Saint-Eustache surent se l'attacher de 1801 à 1805 en lui offrant des appointements de "cinquante louis». Il était surnommé «le maître d'école patriotique" et même si on relevait des déficiences dans ses connaissances grammaticales, il se rachetait et amplement par le zèle et l'application qu'il témoignait à sa profession encore reconnue par la société d'alors, celle de formateur des esprits et des coeurs. ${ }^{4}$

Les études primaires terminées au début d'octobre 1807 , les deux aînés de la famille de Bellefeuille, Eustache-Antoine, âgé de 14 ans, et Louis-Charles de 12, prennent le chemin de Montréal pour y entreprendre le cours secondaire et collégial au Collège de Montréal aussi appelé Petit Séminaire. Il s'agit d'une institution vieille de quarante ans qui vient de s'installer dans un nouveau bâtiment, sis au faubourg SaintAntoine ou des Récollets.

Le Collège de Montréal fondé en 1767 par Jean-Baptiste Curatteau, sulpicien, dans le presbytère de la Longue-Pointe s'était déplacé en 1773 pour occuper le Château Vaudreuil, sis sur l'actuelle place JacquesCartier. Celui-ci fut lourdement endommagé par le feu du 6 juin 1803. La Fabrique propriétaire ayant refusé de fournir un autre bâtiment, le Séminaire construit sur les plans d'Antoine-Alexis Molin, sulpicien (1757-1811), un grand bâtiment au coût de 455481 livres tournois, donc près de 24000 louis. Situé un peu en dehors des murs, à l'ouest de la rue Mc Gill, il pouvait accueillir 120 pensionnaires et plus de 150 externes. ${ }^{5}$ Il comportait un corps central de 198 pieds français de long sur 32 de large (i.e. $210 \times 35$ pieds anglais) et deux ailes de 171 pieds français sur 39 (i.e. $186 \times 43$ pieds anglais). Le bâtiment en

\footnotetext{
${ }^{4}$ Amédée Gosselin : «Louis-Généreux Labadie ou le Maître d'École patriotique» en Mémoires de la Société royale du Canada, 1913, p. 97-124.

${ }^{5}$ Archives du Séminaire de Saint-Sulpice de Montréal (A.S.S.S.M.) S26, cahier 38.
} 
forme de $\mathrm{H}$ possédait une cave, un rez-de-chaussée de 11 pieds, deux étages de 10 et de 9 pieds et une mansarde percée de lucarnes. L'aile ouest logeait une belle chapelle de deux étages à grandes baies, décorée entre autres par Louis Quevillon. C'était donc une imposante construction qui subsista partiellement jusqu'en mai 1981. Le Collège y était resté jusqu'en décembre $1861 .^{6}$

La valeur du Collège provenait de la qualité des prêtres de Saint-Sulpice qui donnaient le ton à la formation que l'on y recevait. Le directeur du Petit Séminaire (on dirait maintenant le supérieur ou le recteur), était assisté surtout de trois autres prêtres d'origine française tous chassés par la Révolution et acceptés au Canada par le gouvernement anglais. Le directeur était Jacques-Guillaume Roque, sulpicien, (1761-1840), ancien professeur du Séminaire d'Angers qui venait d'être nommé supérieur en 1806. Il était assisté par AntoineJacques Houdet (1764-1826), professeur de Philosophie, de Mathématiques et de Sciences, Claude Rivière (1766-1820) qui partageait avec $M$. Houdet les Mathématiques et les cours de lettres en Rhétorique et en Classe d'humanités, et par $M$. Antoine Sattin (1767-1836). Ce noyau de sulpiciens était aidé, occasionnellement, par un prêtre séculier et par quatre ou cinq régents, la plupart du temps des ecclésiastiques qui poursuivaient des études de théologie tout en 'prêtant 'leurs 'services au Collège. En 1809, les régents étaient MM. Jean-Baptiste Saint-Germain, (élève du Collège 1806), Augus McDonald (1806), Nicolas Dufresne, futur sulpicien (1806), et JeanBaptiste Guillon (1809). ${ }^{7}$

Le régime de vie était spartiate. Le coutumier, rédigé par $\mathrm{M}$. Roque, nous le montre. Le lever est fixé à $51 / 2 \mathrm{~h}$, à $53 / 4$ on doit être à la salle d'étude pour la prière du matin suivie de l'étude. À $71 / 4$ déjeûner suivi de la messe à $71 / 2$. Puis il y a classe jusqu'à $10 \mathrm{~h}$. La récréation de 10 minutes précède l'étude. On mange à $11 \mathrm{~h}$. $1 / 4$ et il y a détente jusqu'à $1 \mathrm{~h}$. L'étude de $1 \mathrm{~h}$. est suivie à $2 \mathrm{~h}$. de classes. À $4 \mathrm{~h}$. collation et récréation, puis de nouveau l'étude jusqu'à $61 / 2 \mathrm{~h}$. Chapelet et lecture spirituelle, puis à 7 heures, repas et récréation jusqu'à $71 / 2 \mathrm{~h}$, puis montée au dortoir.

${ }^{6}$ Olivier Maurault, P.A., p.S.S., Le Collège de Montréal 1767-1967, deuxième éd. revue et mise à jour par Antonio Dansereau, p.S.S., Montréal, 1967, p. 21-27. Robert Lahaise, Les édifices conventuels du Vieux Montréal, Cahiers du Québec, Montréal, Hurtubise HMH, 1980, p. 310-322.

${ }^{7}$ A.S.S.S.M. S11,50. Règlement du Petit Séminaire 1806-1860, Archives du Collège de Montréal : Cahier du personnel compilé par M. Antonio Dansereau, p.S.S. 
Et le règlement assigne des places aux élèves au réfectoire, à l'étude et à la chapelle. Il y avait dans tout cela peu de place à la fantaisie et aux caprices du moment. On n'a conservé de cette époque que les palmarès indiquant les noms des élèves ayant mérité des prix. L'aîné des frères de Bellefeuille, Eustache-Antoine, qui n'a été élève que trois ans (1807-1810), connut des succès beaucoup plus brillants que le cadet, Louis-Charles. Cependant le cadet recueillait des accessits durant quatre années en latin et en français, preuve qu'il travaillait bien et semblait profiter des enseignements de ses professeurs.

Le Collège de Montréal conserve aussi deux cahiers qui à mes yeux sont plus précieux que des résultats académiques. Il s'agit d'activité de recherche d'un apostolat dans le milieu qui était le sien. En effet il existait une Congrégation dite du Petit Séminaire aussi appelée Congrégation des Écoliers. Il s'agit d'une congrégation mariale et le premier cahier qui remonte à 1766 a été tenu par M. Jean Brassier (1729-1798), sulpicien. Le mouvement n'est passé au Collège qu'en 1773. On voit que le 5 février 1809, Louis-Charles Lefebvre de Bellefeuille, âgé de 14 ans, est reçu dans cette congrégation. Et à l'autre extrémité du même registre, il devient portier le ler novembre 1814 et préfet le 3 juillet 1814 , poste qu'il occupera jusqu'en novembre suivant. Dans un autre registre, Le Livre de compte de la Congrégation, il signe à titre de préfet le rapport indiquant que les quêtes et les contributions ont rapporté $18 £ 10^{\text {s. Le }} 2$ novembre suivant avant de quitter ce poste, Charles de Bellefeuille remit $33 £ 5^{\text {s }}$ dans les coffres. ${ }^{8}$

M. Louis-Charles Lefebvre de Bellefeuille avait commencé son cours en 1807 avec 73 confrères dont on connaît les noms. Ils appartenaient aux trois divisions du Collège. En Rhétorique en 1812, ils n'étaient plus que 7 élèves. M. de Bellefeuille terminait ses études en 1815, son frère puîné François l'ayant rejoint au nombre des collégiens en 1810 .

${ }^{8}$ Archives du Collège de Montréal, Registre des réceptions des membres de la Congrégation du Petit Séminaire I, 1766-1870, p. 92.

Registre des Élections, p. 3. Livre de compte de la Congrégation, p. 62. 


\section{RÉGENT}

Au début du XIX ${ }^{\mathrm{e}}$ siècle, l'Église du Canada souffrait depuis longtemps d'une pénurie de prêtres. C'était justement au moment où les Collèges se multipliaient, Nicolet en 1803, Saint-Denis en 1805, SaintHyacinthe en 1811, Sainte-Thérèse en 1825. L'aide que l'Église apportait à ces institutions nouvelles ou anciennes était absolument essentielle à leur survie. C'est pourquoi ces maisons comptaient sur les ecclésiastiques pour compléter leur corps professoral. Le Collège de Montréal n'eut qu'un prêtre durant 22 ans, M. Curatteau. En 1794, le nombre atteint 4 et n'a pas augmenté pendant 40 ans. Les régents, comme on les appelait alors, ont reçu une certaine reconnaissance ecclésiale à Montréal lorsque $\mathbf{M}^{\mathrm{gr}}$ Jean-François Hubert, le 12 novembre 1792, approuva un règlement convenable pour ces clercs qui poursuivaient leurs études théologiques tout en remplissant une pleine tâche d'enseignement ou d'animation au Collège. ${ }^{9}$

Les Régents, au Collège de Montréal, étaient choisis par le Supérieur, la plupart des anciens élèves et chargés des premières classes. On en ajoutait deux pour surveiller les études et servir de remplaçant quand un autre professeur s'absentait pour maladie ou autre raison. On les logeait deux par deux, les nourrissait (ils avaient une demi-portion de vin), leur fournissait les objets de première nécessité et leur accordait un traitement de $\mathbf{2 5 0}$ livres tournois par année. Ils se levaient à 5 heures pour prière et oraison. De $18 \mathrm{~h} 30$ à $19 \mathrm{~h}$ ils avaient une lecture spirituelle.

Tout en rendant un service indispensable au Collège, les Régents poursuivaient sous la direction de M. Jacques-Guillaume Roque, sulpicien, ${ }^{10}$ leur formation sacerdotale, suivant des cours que leur donnait le Supérieur, mais aussi étudiant par eux-mêmes sous sa direction.

${ }^{9}$ A.S.S.S.M. S11,30.

${ }^{10}$ Jacques-Guillaume Roque, né à Beaumont,diocèse de Vabres, France, le 24 janvier 1761, mort à Montréal le 2 mai 1840 , devint prêtre en 1785 . Il enseigna au Séminaire d'Angers de 1785 à 1789. Au moment de la Révolution, il était supérieur d'Angers, fut emprisonné et pu gagner en 1790 le diocèse d'Orense en Espagne où il aida au ministère. Il passa aux États-Unis et arriva au Canada le 24 octobre 1796. Fut supérieur du Collège de Montréal de 1806 à 1828 . Il avait été vicaire général sous les évêques de Québec et de Montréal. 


\section{ÉTAPES VERS LE SACERDOCE}

La formation théologique de $\mathrm{M}$. de Bellefeuille progressait, ce dernier franchit les étapes le conduisant à la prêtrise. Le 19 mai 1816, $\mathrm{M}^{\mathrm{gr}}$ Joseph-Octave Plessis le recevait dans le clergé de Québec en la chapelle de l'Hôtel-Dieu en lui conférant la tonsure. ${ }^{11} \mathrm{M}^{\mathrm{gr}}$ BernardClaude Panet lui conférait les quatre ordres mineurs le 30 juin 1817, lors de sa visite pastorale à Saint-Philippe de Laprairie. ${ }^{12} \mathrm{Au}$ début de juin, le même évêque recevait l'engagement définitif de $M$. de Bellefeuille en l'élevant à la dignité de sous-diacre sans cependant avoir noté le fait au registre des ordinations. Pour franchir ce pas, l'Église, selon les prescriptions canoniques du Concile de Trente, s'assure que le futur prêtre aura les moyens de subsistance pour mener une vie décente et ne pas constituer un fardeau pour son diocèse. C'est pourquoi les futurs sous-diacres doivent présenter un titre clérical, c'est-à-dire un engagement lui assurant une rente viagère, qui est proclamé à son église paroissiale comme les bans de mariage. Le 16 mai 1818 sa mère, veuve d'Antoine Lefebvre de Bellefeuille, s'engage par devant Joseph-Amable Berthelot, notaire de Saint-Eustache, à verser un revenu de $150 £$ et 20 sols ou coppies pris sur l'héritage de Louis-Charles lui-même, l'usufruit ayant été laissé à la mère.

Puis le 30 mai 1819 dans l'église de Berthier, $\mathrm{M}^{\mathrm{gr}} \mathrm{Joseph}-$ Octave Plessis l'ordonnait diacre. ${ }^{13}$ Enfin le 5 juin (une semaine après) dans la chapelle de l'Hôpital Général de Montréal des Soeurs Grises, le même évêque l'élevait à la dignité de prêtre. ${ }^{14}$

\section{SAINT-SULPICE, MINISTÈRE À LA PAROISSE}

Le Séminaire de Saint-Sulpice de Paris devenait propriétaire des Seigneuries de l'Isle-de-Montréal et de Saint-Sulpice le 9 mars 1663. Entre les années 1718 et 1735, le gouverneur de Nouvelle-France et le roi de France y ajoutèrent (en trois concessions successives) la Seigneurie du Lac-des-Deux-Montagnes. À la fin du régime français, on a compté en Nouvelle-France jusqu'à 45 Sulpiciens, la grande majorité des prêtres, mais aussi quelques clercs.

\footnotetext{
11 Archives de l'Archidiocèse de Québec (A.A.Q.), H 124 vo.

12 R.A.P.Q. 1933-34, p. 247, lettre de $\mathrm{M}^{\mathrm{gr}}$ Panet à $\mathrm{M}^{\mathrm{gr}}$ Plessis, 30 juin 1817.

13 A.A.Q. H. 227 ro.

14 Ibid., 228 vo.
} 
À la Conquête en 1763, 27 sulpiciens, tous Français, restèrent au Canada, le Séminaire de Paris ayant cédé par acte notarié du 24 avril 1764 ses biens au Séminaire de Montréal. En 1792, il n'y avait plus que huit sulpiciens, deux d'origine française et six Canadiens qui avaient été agrégés avec une certaine hésitation sinon un déplaisir par les survivants français du Canada. Les autorités britanniques prohibaient le recrutement de prêtres en France. La situation fut complètement modifiée avec l'arrivée de 19 prêtres sulpiciens français chassés par la Rẹ́volụtion de 1791 à 1800. Le Séminaire de Saint-Sulpice de Montréal redevint une communauté formée d'une grandé majorité de prêtres d'origine française.

Quand M. Louis-Charles Lefebvre de Bellefeuille commença sa régence au Collège de Montréal en 1815, on comptait 18 sulpiciens : 12 Français et 6 Canadiens. En 1819, il y avait, sur un total de 18, 11 Français, 6 Canadiens et 1 Américain.

En 1835, sur 21 prêtres, 11 Français, 7 Canadiens et trois de langue anglaise pour le ministère auprès des immigrants catholiques d'origine britannique. Il ne fait aucun doute que $M$. de Bellefeuille connaissait et acceptait cet état de chose. Au moment des difficultés du Séminaire avec $\mathbf{M}^{\mathrm{gr}}$ Jean-Jacques Lartigue à Montréal et avec les évêques de Québec, rien n'indique que $M$. de Bellefeuille se soit dissocié de ses confrères.

Encore sous-diacre, à l'été $1818, \mathrm{M}$. de Bellefeuille avait été envoyé à la Mission du Lac-des-Deux-Montagnes avec la mission de s'initier à l'algonquin en transcrivant le dictionnaire que Jean-Baptiste Thavenet, ${ }^{15}$ sulpicien et ancien missionnaire, avait réalisé et qu'il voulait utiliser en France. Il y lia une solide amitié avec M. Anthelme Malard, missionnaire. Par ailleurs il semble qu'une amitié solide s'établit entre $\mathbf{M}$. JeanAuguste-Henry Roux, supérieur du Séminaire de Montréal et M. de Bellefeuille. Il apprécia les qualités de dévouement et de délicatesse de la jeune recrue du Séminaire de Montréal. ${ }^{16} \mathrm{M}$. de Bellefeuille fut agrégé à cette institution le 30 janvier 1821 , après un an de service. ${ }^{17}$ Plus tard, le 31 août 1833, il fut promu au rang des douze assistants.

${ }^{15}$ A.S.S.S.M. Arrivé au Canada en 1794, Thavenet devait en repartir en 1815.

${ }^{16}$ Lettres de Roux à de Bellefeuille, 21 fév., 3 sept., 28 oct. 1825.

${ }^{17}$ A.S.S.S.M. Livre de l'Association des prêtres du Séminaire de Montréal. 
Malgré l'insistance de l'équipe du Lac-des-Deux-Montagnes, $M$. Roux retint $M$. de Bellefeuille au service de la Paroisse pour "qu'il connaisse la maison à laquelle il veut s'attacher». ${ }^{18} \mathrm{M}$. de Bellefeuille effectua trois séjours au Séminaire de Saint-Sulpice de Montréal. Il y travailla de 1819 à 1824. À la mort de M. Boussin, M. de Bellefeuille est appelé à Montréal pour le ministère en anglais. ${ }^{19}$ Puis à la fin de sa vie, de 1834 à 1838, il y fait un troisième séjour. La Paroisse de Montréal s'étendait sur un très grand territoire allant de Verdun et NotreDame de Grâce à l'ouest jusqu'aux confins d'Hochelaga à l'est, du fleuve jusqu'au niveau de la rue Jean-Talon. En 1825, ce territoire regroupait 18133 catholiques sur 26151 habitants. Les fidèles comprenaient 2833 personnes à la campagne, 2776 dans ce qui était autrefois la ville murée et 12524 dans les faubourgs.

Pour desservir ces âmes, le Séminaire offrait de quinze à dix-sept prêtres chargés du ministère des sacrements et du culte dans l'église Notre-Dame, à la chapelle Notre-Dame de Bonsecours et après 1830, à la chapelle des Récollets, centre du ministère auprès des catholiques de langue anglaise et de la Congrégation des hommes. $M$. de Bellefeuille fut responsable de 1819 à 1828 des sépultures et des baptêmes et de la visite des pauvres et des malades des faubourgs et des côtes. Il exerça le ministère dans les faubourgs Saint-Joseph et Saint-Antoine durant plusieurs années et dans les côtes de Verdun et de Saint-Joseph. Le faubourg Saint-Joseph regroupait 2748 habitants et celui de Saint-Antoine 1397. Les côtes qui les bordaient étaient beaucoup moins peuplées cependant. Le prêtre responsable visitait les familles et allait porter les secours de la religion à ceux qui ne pouvaient se déplacer.

À ces tâches s'ajoutaient des sermons à l'église paroissiale. M. de Bellefeuille rendait aussi à l'occasion d'autres services au Séminaire. Il fut réglementaire, receveur du casuel et des messes.

\section{LA MISSION DU LAC-DES-DEUX-MONTAGNES}

M. de Bellefeuille, sous-diacre, avait fait un stage à la Mission du Lac-des-Deux-Montagnes à l'été et à l'automne de 1818. On lui

18 A.S.S.S.M. Lettre de Roux à Malard, 8 oct. 1819.

19 A.S.S.S.M. Lettre de Roque à Roupe, ler juillet 1826. 
avait confié la tâche de transcrire le dictionnaire algonquin de JeanBaptiste Thavenet, sulpicien, missionnaire de 1802 à 1809. Cette tâche lui avait permis de s'initier à la connaissance de cette langue amérindienne. Il avait aussi contracté une solide amitié avec $\mathrm{M}$. Anthelme Malard et avec le directeur de la Mission M. François Humbert, tous deux sulpiciens. C'est sans doute cet attachement qui explique l'insistance de ces deux missionnaires pour recevoir comme collaborateur au Lac M. de Bellefeuille à son entrée à Saint-Sulpice.

En 1824 enfin, M. de Bellefeuille est nommé au Lac-des-DeuxMontagnes comme missionnaire des Algonquins. Il se joint alors à ses deux amis et à Jean-Baptiste Roupe, aussi sulpicien.

La Seigneurie du Lac-des-Deux-Montagnes constituait un domaine de trois lieues et demie de large sur plus de cinq de profondeur. Les Sulpiciens avaient détaché de ces terres une partie de quinze kilomètres de long sur près de dix de profondeur. Ils réservaient ce domaine à l'usage des peuplades amérindiennes.

La Mission, fixée dès 1722 sur la pointe où s'élèvent l'église et le presbytère d'Oka, groupait dès le début deux peuples bien différents, les Iroquois et les Algonquins; les premiers sédentaires et intéressés aux cultures et les deuxièmes encore nomades. Ces derniers quittaient à l'automne la Seigneurie pour passer l'hiver dans les grands espaces du nord et retournaient au printemps à la mission. Le Séminaire de Saint-Sulpice cependant ne donnait aucune concession aux Indiens, il leur accordait l'usufruit d'un lot dans les villages, fournissant même les maisons, et offrait des terres suffisantes pour la culture et l'alimentation des familles. Il leur permettait d'abattre du bois pour leur usage personnel seulement, mais refusait de les laisser exploiter à des fins strictement commerciales le bois du Domaine.

À la suite de la Conquête, les Amérindiens revendiquèrent pour eux la possession de la Seigneurie. Or les titres de concession ne laissaient rien à l'interprétation. La Seigneurie avait été donnée au Séminaire de Saint-Sulpice de Paris, celui de Montréal prenant la succession. Les Amérindiens de la mission constataient pourtant qu'au Sault-Saint-Louis, à Saint-Régis ou à l'Ancienne Lorette, les terres avaient été remises aux aborigènes. Mais il n'en était pas ainsi avec la propriété du Lac-des-Deux-Montagnes. Ce fut l'occasion de revendications véhémentes, au début de la part des Iroquois et ensuite des Algonquins. Les autorités britanniques maintinrent le «statu quo ante». Les tribunaux reconnurent les droits du Séminaire de Saint-Sulpice. 
En 1825, les deux ethnies, les Iroquois et les Algonquins, se groupaient en deux villages. Celui de l'est comptait 355 Algonquins réunis dans 76 maisons ou cabanes. Les Iroquois au nombre de 282 occupaient 56 maisons. ${ }^{20}$ De plus autour des bâtiments de l'église de la mission, il y avait une agglomération regroupant 250 autres personnes. La Mission était desservie par trois missionnaires, le directeur ou supérieur, et un missionnaire pour chaque groupe. Il y avait deux religieuses de la Congrégation de Notre-Dame attachées depuis 1677 à l'éducation des petites indiennes du Fort de la Montagne et qui suivirent la Mission dans ses déplacements.

Les Sulpiciens concédaient depuis 1783 des terres à des colons blancs, mais dans des côtes éloignées du Domaine (Saint-Placide, SaintJoseph du Lac, Saint-Benôit, Sainte-Scholastique). De sorte que les Amérindiens avaient suffisamment de terre pour leurs cultures, de bois pour leur usage et de prairies ( $11 / 2$ mille sur $1 / 2$ mille). Il y avait en plus sur le Domaine des métairies dont la propriété restait au Séminaire, mais que ce dernier louait pour en tirer des profits et un peu pour donner le ton à l'exploitation agricole. C'était cependant source d'ennui pour les missionnaires.

M. de Bellefeuille se destinait aux Algonquins. Il perfectionna sa connaissance de la langue et put aider M. Malard de 1824 à 1826. On possède encore plusieurs sermons en cette langue. Le départ de JacksonJohn Richards, sulpicien, pour l'Europe avec M. Roux, supérieur, détermina le rappel de $\mathbf{M}$. de Bellefeuille à Montréal pour du ministère auprès des catholiques de langue anglaise. Il revint au Lac de 1828 à 1834, M. Malard fatigué et malade ayant été rappelé à Montréal. C'est alors que $M$. de Bellefeuille, à la santé toute fragile, eut l'occasion de donner sa mesure. Car en 1829, il était promu directeur ou supérieur de l'établissement en remplacement de $M$. Jean-Baptiste Roupe. Le supérieur qui était aussi le curé de la paroisse n'avait pas qu'une charge pastorale. Il devait gérer le Domaine de la Mission, prendre en accord avec le procureur de Montréal, M. Joseph Comte, sulpicien, les décisions pour tirer le plus de revenus des métairies, du moulin de la Baie, des locations pour que les secours requis pour les Amérindiens ne grèvent pas trop le budget du Séminaire. Car le Séminaire payait chaque année les frais

${ }^{20}$ Joseph Bouchette, Esq. A Topographical Dictionary of the Province of Lower Canada, 3 vol., London, Longman, Rees, Orme, Brown, Green and Longmann, 1832, vol. 3, Lake of Two Mountains. 
des Religieuses de la Congrégation de Notre-Dame, institutrices des jeunes filles. Il défrayait aussi la pension des trois Sulpiciens attachés à la Mission et une multitude de services pour les Indiens.

On relève dans la correspondance son souci de défendre la foi de ses fidèles sollicités par des ministres protestants qui distribuaient des bibles. Il y eut aussi des épidémies qui décimaient les Indiens. Les autorités lui demandaient de trouver des médecins qui soigneraient les malades même si le gouvemement ne défrayait pas les dépenses. ${ }^{21}$

Les relations avec les Indiens n'étaient pas toujours faciles. Les archives conservent une plainte du 15 juillet 1828, au Colonel D.F. Napier par quelques Algonquins. ${ }^{22}$ Le factum énumère dix chefs d'accusation contre $\mathbf{M}$. de Bellefeuille surtout centrés sur une vieille femme objet de reproches et sur des spoliations subies par d'autres Indiens. Après des réunions du conseil, les membres désavouent les personnes qui ont porté des accusations et constatent l'absence de raisons valables pour la démarche contre les Sulpiciens.

Après trois séjours à la Mission du Lac-des-Deux-Montagnes (automne $1818,1824-26$ et 1828-34), $M$. de Bellefeuille est rappelé au Séminaire de Saint-Sulpice de Montréal pour vaquer au ministère pastoral de la Paroisse de Notre-Dame de Montréal.

\section{LES VOYAGES AU TÉMISCAMINGUE ET EN ABITIBI (1836-1838)}

\section{a) Origine}

Le diocèse de Montréal venant d'être érigé, $\mathrm{M}^{\mathrm{gr}} \mathrm{J}$ ean-Jacques Lartigue donna une impulsion plus grande à l'action missionnaire aux confins du diocèse. L'évêque avait déjà demandé en 1832 à $\mathrm{M}$. Vincent Quiblier l'envoi de M. Flavien Durocher pour établir la présence de l'Église dans le nord-ouest du Québec, mais en vain. ${ }^{23} \mathrm{M}$. Roupe avait bien exécuté de 1815 à 1828 des voyages sur le bas Outaouais et, deux fois par année, séjournait trois semaines dans la région de Montebello.

\footnotetext{
${ }^{21}$ A.S.S.S.M. Lettre du docteur Arthur Stewart à de Bellefeuille, 13 septembre, 17 septembre et 20 novembre 1832 .

${ }_{22}$ A.S.S.S.M. Cahier no 1 de Joseph Isidore Tallet, p.S.S.

${ }^{23}$ R.A.P.Q. 1942-43, p. 145, $\mathrm{M}^{\mathrm{gr}}$ Lartigue à $\mathrm{M}^{\mathrm{gr}}$ Panet, 12 mai 1832.
} 


\section{b) Mission du Témiscaming (20 juin - 16 août 1836)}

Le 29 mai 1836, $\mathrm{M}^{\mathrm{gr}}$ Lartigue écrit que "La mission de Témiscaming est enfin résolue par décision du Docteur Stevenson qui déclare M. de Bellefeuille assez fort pour aller dans cette terre infidèle». ${ }^{24}$ Le choix de l'évêque se porta sur $\mathbf{M}$. de Bellefeuille parce qu'il parlait couramment l'algonquin et l'anglais. On lui adjoignit $M$. Jean-Baptiste Dupuy, professeur de théologie à l'école de l'Évêché. ${ }^{25} \mathrm{M}^{\mathrm{gr}}$ Lartigue leur transmit de Longueuil les instructions suivantes qui se résumaient ainsi :

«Il leur donne mission de prendre possession de cette terre infidèle au nom de l'Église catholique, d'y examiner l'état de la population des sauvages et leurs dispositions à embrasser le christianisme. Ils suivront autant que possible la ligne de conduite suivante : $1^{\circ}$ ils planteront une croix sur le site de leur future chapelle dont le patron sera saint" Adalbert, évêque de Prague et martyr, apôtre des Prussiens, des Hongrois et des Polonais, puis ils parcourront en surplis et la clochette à la main la bourgade des sauvages pour leur annoncer qu'ils sont les envoyés du seul vrai Dieu; $2^{\circ}$ ils enseigneront à quelques jeunes gens intelligents l'abrégé de la doctrine chrétienne pour servir de catéchistes capables d'instruire les autres et de baptiser au besoin; $3^{\circ}$ le baptême ne sera guère donné qu'aux moribonds, suivant la doctrine de saint Liguori; $4^{\circ}$ les missionnaires auront sur les sauvages d'au-delà du township de Hull les pouvoirs spirituels que leur attribue le mandement de 1793, ainsi que ceux des archiprêtres et des curés concernant les mariages en particulier; $5^{\circ}$ ils s'informeront des principaux vices des sauvages et si les blancs leur vendent de l'eau-de-vie; $6^{\circ}$ ils apprendront l'état des sauvages, leur nombre, leur langue, leurs causes, leurs rapports avec la Compagnie de la Baie d'Hudson; ils tiendront aussi un journal de leur mission, et ils transmettront les noms de ceux qu'ils auront choisis pour baptiser, marier et catéchiser. $\mathbf{M}^{\mathrm{gr}}$ Lartigue donne aussi aux deux missionnaires les pouvoirs ordinaires de cure en faveur des diocésains du Haut-Canada et du district épiscopal du Nord-Ouest. " ${ }^{26}$

${ }^{24}$ Ibid., 1944-45, p. 191, I. Bourget, secrétaire de $M^{\text {gr }}$ Lartigue, à P. Brunet, 29 mai 1836. Sur la Mission du Témiscaming et du Lac Abitibi, voir Yvon CHARRON, p.S.S., «Monsieur Charles de Bellefeuille, missionnaire de l'Outawais (1836-38)», dans Revue d'Histoire de l'Amérique française, vol. V, no 2, sept. 1951, p. 193-226.

25 Jean-Baptiste Dupuy, né à Contrecoeur en 1804, ordonné prêtre en 1832, professeur de théologie à l'évêché de Montréal en $1835-1836$, vicaire à Chambly et directeur du Collège en 1836, rédacteur des Mélanges religieux en 1843-1844, directeur du Collège de l'Assomption de 1846 à 1852, curé d'Iberville de 1852 à 1858 et de SaintAntoine-sur-Richelieu de 1858 à 1877 où il décéda en 1879.

${ }^{26}$ Ex. Pièces et Actes, t.2 f.150 r de Mgr Lartigue dans R.A.P.Q. 1944-45, p. 192. 
M. Dupuy part le 20 juin de Lachine sur un petit bateau à vapeur. L'après-midi, M. de Bellefeuille rejoint le groupe à la Mission du Lac-des-Deux-Montagnes. En trois jours de navigation, le «steamboat" atteint les rapides aux Chats, 10 lieues à l'ouest d'Aylmer. Le canot prévu à cet endroit n'est pas au rendez-vous. Mais on embauche trois aides au salaire de 15 à 18 piastres par mois. Avec ces hommes, M. Dupuy va chercher à Fort Coulonge l'esquif nécessaire et y trouve aussi noürititure et foumiture pour co voyage. Et pendant ce temps, M. de Bellefeuille prêche dans les environs et fait des conversions, des mariages et chez les blancs et chez les Amérindięns. Les quatorze premiers jours de juillet se passent à pagayer, camper et s'efforcer de gagner Témiscaming où ils arrivèrent le 14 juillet à 4 heures, au poste sis à 450 milles de Montréal. Les missionnaires sont très bien reçus par les autorités de la Compagnie de la Baie d'Hudson, même si les Indiens nomades ont quitté apeurés par la nouvelle de la venue des prêtres. Les treize jours qui suivent s'écoulent à des labeurs apostoliques, les Indiens au nombre de 220 participant aux diverses cérémonies dont celle de la bénédiction d'une croix, site de la chapelle de Saint-Adalbert, le 19 juillet.

Le 27 juillet, les Missionnaires doivent s'éloigner car il n'y avait plus de vivres pour nourrir la foule des nomades venus entendre la parole du Christ. Le groupe reprit le chemin du retour et le 16 août 1836, M. de Bellefeuille arrivait à Montréal, après un mois et 26 jours de voyage. Cette première expédition avait donné 142 nouveaux chrétiens, 19 adultes et 123 enfants, 28 personnes avaient fait leur première communion et on avait donné le sacrement de mariage à 4 couples.

\section{c) Mission du Lac Abitibi (7 juin - 23 août 1837)}

Les résultats du voyage de 1836 étant la preuve du succès de la mission, M. de Bellefeuille accepte de refaire un autre essai èn 1837. Il est seul prêtre accompagné de quatre hommes dans un canot de 8 à 9 rames, chargé de provisions et de bagage. Parti de Lachine le 7 juin 1837 , le canot atteignit le Fort Témiscaming le 24 suivant. M. de Bellefeuille s'efforce de développer le sens chrétien des nouveaux baptisés de l'année précédente, reçoit la confidence du bourgeois, chef de poste, Monsieur Cameron, comme quoi son influence a été bénéfique. Après quinze jours de séjour, $M$. de Bellefeuille poursuit sa route vers le grand lac Abitibi, car il n'y avait pas moyen de nourrir les 300 bouches regroupées. Du 9 au 12 juillet, on parcourt les 50 lieues qui séparaient Témiscaming du Fort Abitibi. Après un accueil froid, l'arrivée du bourgeois $\mathrm{M}$. Fraser changea completement les dispositions et des Blancs 
et des Amérindiens. Le poste est modeste (deux maisons et deux hangars). Le missionnaire put exercer son ministère durant neuf jours, baptisant 32 enfants et 12 adultes. Il repartit même avec les deux enfants d'un commis de la Compagnie, un métis, qui voulait les voir instruire et baptiser à la Mission du Lac-des-Deux-Montagnes. Au retour, il s'arrêta six jours à Témiscaming où il baptisa $\mathbf{4 5}$ autres personnes dont 34 adultes et présida à deux mariages.

Au début d'août, $M$. de Bellefeuille quitta Témiscaming pour aller exercer son ministère au Fort aux Allumettes, atteint en quatre jours par un parcours de 85 lieues. L'année précédente, on n'avait fait qu'une très courte halte d'un jour à cet endroit. Il y séjourne alors 10 jours pour les 15 ou 16 familles sauvages du poste. Après avoir baptisé 19 adultes sauvages, 8 enfants indiens, 9 enfants irlandais et un écossais, béni 6 mariages, il reprit le chemin du retour le 23 août et revint à Montréal après deux mois et demi d'absence.

M. de Bellefeuille avait baptisé 90 enfants et 100 adultes, présidé 21 mariages et fait faire la première communion à 34 personnes.

\section{d) Mission du Grand Lac Victoria (29 mai - 8 septembre 1838)}

M. de Bellefeuille entreprit enfin une troisième mission en 1838. Il partit de Lachine le 29 mai 1838, accompagné de six hommes et de bagage. Pendant cinquante jours de marche, il parcourut dans un véhicule fort fragile quinze cents milles et passa cent trente-six rapides. Arrivé le 15 juin à Témiscaming, il y passa 25 jours pour affermir dans la foi les fidèles et compléter la chapelle commencée deux ans avant. Il baptisa 38 personnes dont 26 adultes. Puis le 11 juillet, il s'embarqua pour le lac Abitibi, atteint en six jours, où il fut reçu avec grande joie. Il y avait 150 sauvages qui s'étaient réunis. Le 25 juillet « fatigué à n'en pouvoir plus et pensant que quelques jours de voyage accommoderaient mieux ma santé que l'ouvrage de la Mission, je me décidai à partir pour le Grand Lac». ${ }^{27}$ Il s'agit de revenir vers le sud et de prendre vers l'est la Rivière des Quinze menant au Grand Lac Victoria. Ce dernier se trouve aujourd'hui dans le parc La Vérendrye. Arrivé le 2 août, il

${ }^{27}$ Rapport de l'Association de la Propagation de la Foi, Montréal, juin 1840, no 2, p. 82. Le "Journal d'un voyage fait à Témiskaming en 1836 par J.-B. Dupuy, ptre" a été publié dans le Rapport, no 1 , mai 1839 , p. 24 à 53. La «Relation d'une Mission faite à l'été 1837 et Précis de la relation de la troisième mission par M. de Bellefeuille, p.S.S. » dans le Rapport, no 2, juin 1840, p. 17 à 88. 
rassembla de 40 à 50 sauvages pour une mission de six jours. Il baptisa sept adultes et quatorze enfants. Le retour commença le 8 août. Il arrêta cinq jours à Témiscaming baptisant 14 autres adultes et prenant les dispositions pour faire finir la chapelle. Enfin le 20 août 1838, il quitta définitivement ce poste qui lui tenait tant à coeur pour arriver à Montréal le 8 septembre 1838, ayant été absent 100 jours.

\section{QUELQUES REMARQUES}

De 1836 à 1838, la partie habitée à l'ouest de Montréal s'étendait de Montréal jusqu'à Fort aux Allumettes. On rencontrait ici et là des familles de Blancs et d'Amérindiens installées dans des maisons ou des campements, attirées par le commerce du bois ou la possibilité de vivre de chasse et de pêche.

Il y avait plusieurs catholiques dans cette population négligée alors par l'Église à cause surtout des accidents géographiques. M. de Bellefeuille y a rencontré beaucoup de Sauvages qu'il avait déjà connus au Lac-des-Deux-Montagnes. Et tous manifestaient une joie et une confiance non suspecte au missionnaire et à son ministère.

L'honorable Compagnie d'Hudson manifestait une certaine sympathie au missionnaire et à son action. Ses employés, sauf un commis de second ordre au Lac Abitibi, accueillirent avec beaucoup de chaleur le visiteur. La Compagnie prêta aussi un canot et facilita l'acquisition de marchandises nécessaires au voyage, le prix de ces dernières étant cependant défrayé par l'Église.

Les voyages étaient cependant périlleux et inconfortables. Lors du premier, un des rameurs originaire de Sorel fut tué, la tête écrasée contre un rocher par le canot. $M$. de Bellefeuille, aidé par un Indien pour traverser un cours d'eau, tomba aussi à l'eau une fois. Il fut aussi très incommodé par les moustiques.

Les Indiens, clairsemés, vivaient dans une pauvreté totale. Ils ne connaissaient pas d'organisation sociale bien étroite, la recherche de subsistance les éloignant les uns des autres. Ils étaient sympathiques aux missionnaires, bien que adonnés à la polygamie, à l'ivrognerie et à la paresse, ce qui aggravait leur extrême pauvreté. 
De 1836 à 1838, M. de Bellefeuille avait parcouru dans des conditions on ne peut plus pénibles approximativement 3500 milles sur l'Outaouais, prêché, administré le baptême à environ 550 personnes, donné les sacrements dans des dizaines de villages ou campements. C'étaient là des activités propres à éreinter les personnes jouissant d'une solide santé. Mais comment $\mathbf{M}$. de Bellefeuille a pu entreprendre ces tâches relève du mystère; toute sa vie il eut une santé très frêle.

M. Jean-Joseph-Auguste Roux, supérieur, écrivait le 8 octobre 1819 à $\mathbf{M}$. Arthème Malard, "Nous vous priverons quelque temps de $\mathbf{M}$. de Bellefeuille mais pour vous le rendre ensuite si sa vocation et sa santé le permettent." ${ }^{28} \mathrm{M}$. Roux écrivait à $\mathrm{M}$. de Bellefeuille au Lac-des-Deux-Montagnes le 21 février 1825 : «Adoucissez votre carême et Suivez les conseils de Mr Humbert : Vous savez comme votre Santé nous est chère, comme votre conservation est précieuse à mon coeur." 29 Vers 1827, M. de Bellefeuille écrivait à M. Candide-Michel Le Saulnier, p.S.S., curé de Notre-Dame : "Quand [sic] à Mr Richard, je suis mortifié de ne pouvoir moi-même faire l'ouvrage qu'il demande, ma mauvaise santé, mon état de faiblesse continuelle m'a obligé de suspendre un pareil travail que $\mathrm{j}$ 'avais entrepris.» ${ }^{30}$

Le 29 mars 1830, M. de Bellefeuille écrit à M. Joseph Comte, p. S.S., procureur : «Je vous quitte pour écrire à Mr Humbert, ce sera la $4 \mathrm{e}$ lettre après dîner, c'est bien assez pour un pauvre valétudinaire comme moi. " ${ }^{31}$

En juin suivant au même, il pouvait confier : «Pour ma santé, je suis assurément un peu mieux, mais je n'ai toujours pas grandes forces et la moindre application surtout d'écrire ne fusse qu'une petite lettre me fatigue toujours considérablement. " ${ }^{32}$

M. Joseph-Vincent Quiblier, p.S.S.., faisait part à $\mathbf{M}^{\mathrm{gr}}$ Jean-Jacques Lartigue, évêque de Montréal, le 5 mai 1832 : «Aujourd'hui, nous avons

${ }^{28}$ A.S.S.S.M. Correspondance de Roux.

${ }^{29}$ Ibid., Corr. de Roux.

30 Ibid., Corr. de Bellefeuille.

31 Ibid., Corr. de Bellefeuille.

32 Ibid., Corr. de Bellefeuille. 
appris que Mr de Bellefeuille retenu au lit depuis plusieurs semaines avait enfin un petit mieux. Comme les malades et les convalescents partagent la vie de ce cher confrère, il y aurait imprudence, cruauté et même impossibilité de le charger de l'oeuvre des Algonkins." ${ }^{33}$

\section{MORT LE 25 OCTOBRE 1838}

Revenu du troisième voyage le 8 septembre 1838 , M. de Bellefeuille fit un baptême le 13 septembre et présida à un mariage le 15 septembre de la même année. Selon la notice nécrologique, il ne fut malade que huit jours de fièvres typhoïdes. Il mourut le 25 octobre 1838 et fut enseveli dans une voûte sous la basilique actuelle de Notre-Dame de Montréal le 27, par $\mathrm{M}^{\mathrm{gr}}$ Ignace Bourget, alors évêque coadjuteur de Montréal, assisté de MM. Jean-Baptiste Roupe et Claude Fay, deux sulpiciens. M. de Bellefeuille était âgé de 43 ans, neuf mois et treize jours. Les restes, réunis à ceux d'autres sulpiciens, reposent depuis 1913 sous la chapelle du Grand Séminaire de Montréal.

\section{CONCLUSION}

M. Louis-Charles Lefebvre de Bellefeuille a touché les âmes et les coeurs dans tous les milieux où il exerça son apostolat. Le témoignage le plus frappant est celui du P. Laverlochère, oblat, qui écrivait : "Il fallait que le premier missionnaire de ces lieux eût un talent singulier pour s'attacher les coeurs. Bien qu'il y ait déjà sept ans que Dieu l'a rappelé à lui, son nom est encore béni de tous ceux qui l'ont connu, de quelque origine et de quelque religion qu'ils soient." ${ }^{34}$

\section{J.-Bruno HAREL, p.S.S., archiviste, Séminaire de Saint-Sulpice, Montréal.}

\footnotetext{
${ }^{33}$ Ibid., Corr. de Quiblier.

${ }^{34}$ Donat MARTINEAU, Le Fort Témiskaming, Rouyn, S.S.J.-B. de l'Ouest Québécois, 1979, p. 65. Soeur PAUL-ÉMILE, s.g.c., Baie James, 300 ans d'Histoire, p. 76-77.
} 